\title{
Lattice constant measurement from electron backscatter diffraction patterns
}

\author{
Saowadee, Nath; Agersted, Karsten; Bowen, Jacob R.
}

Published in:

Journal of Microscopy

Link to article, DOI:

10.1111/jmi.12529

Publication date:

2017

Document Version

Peer reviewed version

Link back to DTU Orbit

Citation (APA):

Saowadee, N., Agersted, K., \& Bowen, J. R. (2017). Lattice constant measurement from electron backscatter diffraction patterns. Journal of Microscopy, 266(2), 200-210. https://doi.org/10.1111/jmi.12529

\section{General rights}

Copyright and moral rights for the publications made accessible in the public portal are retained by the authors and/or other copyright owners and it is a condition of accessing publications that users recognise and abide by the legal requirements associated with these rights.

- Users may download and print one copy of any publication from the public portal for the purpose of private study or research.

- You may not further distribute the material or use it for any profit-making activity or commercial gain

- You may freely distribute the URL identifying the publication in the public portal

If you believe that this document breaches copyright please contact us providing details, and we will remove access to the work immediately and investigate your claim. 


\title{
LATTICE CONSTANT MEASUREMENT FROM ELECTRON BACKSCATTER DIFFRACTION PATTERNS
}

\author{
N. Saowadee*, K. Agersted, J.R. Bowen ${ }^{\dagger}$ \\ Department of Energy Conversion and Storage, Technical University of Denmark, Risø Campus, \\ Frederiksborgvej 399, 4000 Roskilde, Denmark.
}

Key words: EBSD, EBSD pattern, Kikuchi band width, Lattice constant, strontium titanate, XRD, Yttria stabilized zirconia.

\begin{abstract}
Kikuchi bands in election backscattered diffraction patterns (EBSP) contain information about lattice constants of crystallographic samples that can be extracted via the Bragg equation. An advantage of lattice constant measurement from EBSPs over x-ray diffraction (XRD) is the ability to perform local analysis. In this study lattice constants of cubic STN and cubic YSZ in the pure materials and in co-sintered composites were measured from their EBSPs acquired at $10 \mathrm{kV}$ using a silicon single crystal as a calibration reference. The EBSP distortion was corrected by spherical back projection and Kikuchi band analysis was made using in-house software. The error of the lattice constant measurement was determined to be in the range of $0.09-1.12 \%$ compared to values determined by XRD and from literature. The confidence level of the method is indicated by the standard deviation of the measurement which is approximately $0.04 \AA$. Studying Kikuchi band size dependence of the measurement precision shows that the measurement error decays with increasing band size (i.e. decreasing lattice constant). However, in practice the sharpness of wide bands tends to be low due to their low intensity, thus limiting the measurement precision. Possible methods to improve measurement precision are suggested.
\end{abstract}

\footnotetext{
* Now at Physics Department, Faculty of Science, Khonkean University, Khonkean 40002, Thailand

† Corresponding author: J.R. Bowen. Tel: +45 4677 4720; email: jrbo@dtu.dk
} 


\section{Introduction}

Lattice constants of crystalline materials are generally measured using the technique of X-ray diffraction (XRD) (Cullity and Stock, 2001) or neutron diffraction (Furrer et al., 2009). The measured lattice constant using XRD is an average value over the whole or large portions of the sample and thus many grains in a polycrystalline sample. For sparsely populated secondary phases, XRD peaks may not appear with sufficient intensity above the background signal and in such cases determining the lattice constant of minor phase fractions is impossible by XRD. Electron backscatter diffraction (EBSD) is a technique principally used for measuring the lattice orientation of crystalline samples in the scanning electron microscope (SEM) by collecting electron diffraction patterns that originate from the local position of the electron beam (Schwartz, 2009). The patterns contain Kikuchi bands whose width in the EBSP contain information directly related to their specific lattice plane spacing by the Bragg equation (eq. 1). Therefore, knowing the band width and the crystal plane of the band allows lattice constant determination from EBSPs. Since the EBSP is obtained locally in the SEM, lattice constant measurement of crystals of minor phases can be performed assuming that they can be located by e.g. SEM image contrast or elemental analysis, and subsequently crystallographically indexed. However, although this is theoretically and experimentally straight forward, the precision of measuring lattice constants from EBSPs needs consideration.

Early efforts to automate pattern indexing (Wright and Adams, 1991) recognised precision limitations of using detected band widths. Thus during automated analysis, EBSPs are crystallographically indexed by comparing angles between detected bands to look-up tables of known crystallographic data.

EBSPs have previously been used to analyse crystallographic parameters. Wilkinson used normalised cross-correlation to detect small shifts in zone axis position in high angular resolution EBSPs (Wilkinson, 1996). The zone axis shift in SiGe eptixaial layers on Si substrates was used to calculate biaxial lattice strain to $0.02 \%$ as zone axis shift in this case implied a cubic-tetragonal distortion. This method has been adapted to low angular resolution wide angle EBSPs to determine 
lattice strain with equivalent accuracy to high resolution EBSPs (Wilkinson et al., 2006). However, without knowledge of Kikuchi band widths hydrostatic changes in lattice parameter remain inaccessible. Michael and Eades used higher-order Laue zone (HOLZ) rings to measure lattice plane spacing in the seven basic crystal systems with deviations to calculated values in many cases to $<1 \%$ (Michael and Eades, 2000). They also demonstrate that multiple HOLZ ring measurements within EBSPs can be reproducible to $0.003 \mathrm{~nm}$. Keller et al. used pattern quality measurements as an indirect method to detect changes in lattice parameter to determine the elastic strain field around $80 \mathrm{~nm}$ thick oxidised AlGaAs layers sandwiched between GaAs layers on a GaAs substrate (Keller et al., 2004). Measurement of EBSP pattern quality provides a scalar quantity reflecting Kikuchi band broadening associated with a range of lattice spacing in the electron beam interaction volume induced by lattice strains averaged over all lattice planes. In a thorough presentation of the advantage of spherical EBSPs, Day demonstrated how average Kikuchi band profiles can be extracted by projecting the sphere to a cylinder normal to the band (Day, 2008). Alternatively band widths can be elegantly extracted by inversion of the spherical map that effectively integrates bands to disks of diameter equivalent to the band width. Although not the focus of the work, the information naturally yields lattice constants. Recently band profiles have been used to determine chirality (Winkelmann and Nolze, 2015) and, Ram et al. have developed a rigorous “Kikuchi bandlet” method to deconvolute intersections of overlapping Kikuchi bands to improve band edge detection to better than $0.1^{\circ}$ in EBSPs (Ram et al., 2014). Dingley and Wright (Dingley and Wright, 2009) used information from EBSPs to determine unit cell space group and lattice constants of silicon and titanium crystalline samples. Li and Han reported a method using a large number of manually identified bands from a single EBSP to determine the Bravais lattice of an unknown mineral with lattice parameter accuracy of $4 \%$ and $1.1^{\circ}$ (Li and Han, 2015). These latter two works however focused on determination of unit cell space group rather than the precision of lattice constant calculation.

In this work, we focus on studying the accuracy of measuring of lattice constant from EBSPs and compare the results to the values measured by XRD. Materials used in this study are composite 
samples of doped strontium titanate (STN) and yttria-stabilised zirconia (YSZ). Both crystalline have cubic lattices but have different lattice constants. STN and YSZ were chosen in this study because they are used in development of solid oxide fuel cell and electrolysis cell electrodes in our research group. Some background and application of STN and YSZ in solid oxide fuel cells can be found in (Sudireddy and Agersted, 2014).

\section{Computing lattice constants from Kikuchi band widths}

In EBSP, Kikuchi bands are formed by backscattered electrons that satisfy the Bragg equation:

$$
2 d_{h k l} \sin \theta=n \lambda
$$

where $d_{h k l}$ is lattice spacing of ( $\left.h k l\right)$ planes, $\theta$ is Bragg angle, $\lambda$ is electron wavelength and $n$ is order of interference (in this case $n$ is 1). Figure 1 illustrates schematic of projection of backscattered electron on a phosphor screen in the SEM chamber. Spherical projection of the diffraction pattern on a flat phosphor screen yields distorted Kikuchi bands. Consequently bandwidths $\left(W_{h k l}\right)$ of the bands located far from the pattern center are wider than usual. To obtain the correct bandwidth $\left(A_{h k l}\right)$ of the projection at the detector distance $(D)$, the flat EBSP has to be radially back projected to the spherical surface of radius $D$ as shown in Figure 1. See Appendix 1 for a description of the back projection method. The correct bandwidth $A_{h k l}$ relates to the Bragg angle (in radius) as equation 2

$$
A_{h k l}=2 \theta D
$$

Since the Bragg angle is very small, the term $\sin \theta$ in equation 1 can be replaced by $\theta$. Substitution of $\theta$ in to equation 2 yields

$$
d_{h k l}=\frac{\lambda D}{A_{h k l}}
$$

Equations 4 and 5 (Kelly et al., 2000) are the relationship of lattice constant (a) of a cubic lattice and lattice spacing $d_{h k l}$ of plans $h k l$ and lattice constants $(a, c)$ of tetragonal lattice and lattice spacing $d_{h k l}$ of plans $h k l$ respectively.

$$
\text { (Cubic) } \quad \frac{1}{d_{h k l}^{2}}=\frac{h^{2}+k^{2}+l^{2}}{a^{2}}
$$




$$
\text { (Tetragonal) } \quad \frac{1}{d_{h k l}^{2}}=\frac{h^{2}+k^{2}}{a^{2}}+\frac{l^{2}}{c^{2}}
$$

The relationship for other lattice systems can be found in for example (Koch, 2006). For cubic structures, the lattice constant $a$ can be obtained by substituting $d_{h k l}$ from equation 3 into equation 4. In practice the product $\lambda D$ can be treated as a calibration constant $(C=\lambda D)$ resulting final equation for compute the lattice constant (eq.6).

$$
a=\frac{C}{A_{h k l}} \sqrt{h^{2}+k^{2}+l^{2}}
$$

A well-known lattice constant sample can be used to find the calibration the constant $C$. In this work only cubic lattices were studied. Tetragonal or more complicated systems have two or more unknown parameters and thus an advanced method of data fitting is needed to solve for the lattice constants.

\section{Experimental}

\section{Materials and sample preparation}

Four samples used in this experiment are $\mathrm{Sr}_{0.94} \mathrm{Ti}_{1.0} \mathrm{Nb}_{0.1} \mathrm{O}_{3}$ (STN94) sintered at $1450{ }^{\circ} \mathrm{C}$ in air, 8 mol.\% yttria-stabilised zirconia (8YSZ), a dense binary mixture of STN94 and 8YSZ (50:50) sintered in gas mixture 9 vol.\% hydrogen and 91 vol.\% argon (STN-YSZ 50\% noxal9) and a dense binary mixture of STN94 and 8YSZ (90:10) sintered in air (STN-YSZ 10\% air). The two binary mixtures were selected in this study since an STN lattice constant dependency on sintering atmosphere was reported by Karczewski (Karczewski et al., 2010). Therefore, potential of measurement lattice constants from EBSPs to differentiate their lattice constants was investigated. A summary of the four samples is shown Table 1.

The 8YSZ sample was carbon coated with an approximate thickness of 10-18 nm to eliminate surface charging in SEM analyses. The sample surfaces were milled with a 30kV $2 \mathrm{nA}$ focused ion beam (FIB) approximately $1 \mu \mathrm{m}$ in depth to remove residual stress and damage from mechanical polishing. The milled surfaces were then polished with a FIB 5kV 2.5 nA probe to remove the damage 
layer created by the $30 \mathrm{kV}$ FIB milling. More detail of sample surface preparation by FIB polishing can be found in (Saowadee et al., 2013). A silicon single crystal was used as a known reference to calculate a calibration constant. The silicon sample surface was prepared in the same manner as the four ceramic samples.

\section{EBSP acquisition}

EBSD work was performed on a CrossBeam 1540XB ${ }^{\mathrm{TM}}$ (Zeiss, Oberkochen Germany) equipped with a Nordlys STM EBSD detector (Oxford Instruments, High Wycombe UK). The Flamenco program within the CHANNEL 5 software suite (Oxford Instruments, High Wycombe UK) was used for collection of EBSPs and indexing Kikuchi bands. EBSD acquisition parameters used were pixel Binning 1x1, gain amplification low, Frame averaging 20 and integration time is on the order of 300 -1000 ms. An SEM working distance of 15 mm was used. Two sets of EBSPs from each sample were collected. The first set was acquired at $30 \mathrm{kV}$ and EBSD detector distance $17.727 \mathrm{~mm}$. This data set was used for indexing Kikuchi bands in the EBSPs. The second set was collected at SEM $10 \mathrm{kV}$ and EBSD detector distance $35.527 \mathrm{~mm}$. Detector distances were determined from pattern centre calibration parameters output as a ratio to the EBSP width. The second set yields a higher magnification of the EBSP of the first set. The latter data set was used for measuring Kikuchi bandwidths to maximize the measurement precision. However, the first set is needed for optimum Kikuchi band indexing. A summary of EBSPs data collection is listed in Table 2.

\section{Lattice constant calculation}

There are four main steps to measure lattice constant from EBSPs:

1) Indexing EBSPs

Each EBSP collected at SEM $30 \mathrm{KV}$ was carefully indexed using the CHANNEL5 Flamenco software. To enable indexing, match units of STN and YSZ were created using the Twist program of Channel 5 software suite based on crystallographic information proposed by Page et.al. (Page et al., 2008) and Yashima et.al. (Yashima et al., 1994). The space group and lattice constant of STN is 
$p m \overline{3} m$ and $3.9237 \AA$ respectively and correspondingly for YSZ is $F m \overline{3} m$ and 5.14086 $\AA$. Match units of Si were created using space group $F d \overline{3} m$ and its standard lattice constant $a=5.43053 \AA$ (Többens et al., 2001). Pattern indexing yields the zone axes in the EBSP required to determine the Kikuchi band Miller indices.

2) Determination of Miller indices of Kikuchi band.

Five widest observable bands in each EBSP were selected. Miller indices of the selected bands were determined by comparing the EBSP with a simulated EBSP generated by using the HKL Fast Acquisition software (Oxford Instruments, High Wycombe, UK). The zone axes resulting from step 1 were used in the comparison manually. After matching the zone axes to find the orientation of the EBSP, the Miller indices of the selected bands can be determined.

3) Bandwidth measurement

The correct bandwidth $A_{h k l}$ of the selected bands in each EBSP was measured using an inhouse developed program “EBSP-Geo” using Microsoft Visual C++ 2008 Express edition (Saowadee, 2014). For our system the full EBSP image is 1344 x 1024 pixels corresponding to the phosphor screen size of $38 \times 28 \mathrm{~mm}$, which gives a sensor pixel size of $0.02827 \mathrm{~mm}$. This constant is used to convert distance units between pixels and $\mathrm{mm}$ in the calculation of the software. The final measured band width is in pixels. Figure 2 shows a distortion corrected band of STN in STN94 by the radial back projection method (Saowadee, 2013), its row average intensity profile (black line) and its slope (blue line). The edges of the band are defined as the positions of maximum and minimum slope. The edges were manually located. The advantage of this method compared to local band width measuring is that it integrates an entire band and thus gives a more statistically robust measure of band width. Naturally longer Kikuchi bands in the EBSP give a better statistical value. In this experiment the length of Kikuchi bands is in the range 396-1136 pixels. A potential source of error of this method is horizontal alignment of the band. Band misalignment results in blurring of the intensity slope and thus leads to loss of precision in the position of the maximum and minimum. In this work Kikuchi bands were aligned manually to obtain the sharpest slope profile. 
4) Lattice constant computing

The final step is to compute lattice constants of STN and YSZ in each sample using equation 6. Before utilising equation 6 to compute lattice constant, the calibration constant has to be determined.

\section{Calibration constant}

The band widths of five crystal planes in Si were used to compute the calibration constant $C=\lambda D$ using equation 6 where $a=5.43053 \AA$. The bandwidth $A_{h k l}$ and Miller indices of the selected bands can be determined as described in the previous section.

\section{Reference lattice constant of STN and YSZ}

Standard lattice constants of cubic STN for STN94 (Page et al., 2008) and cubic YSZ for 8YSZ (Yashima et al., 1994) were used as a reference in this study. The lattice constants of cubic STN and YSZ in STN-YSZ 50\% (noxal9) and STN-YSZ 10\% (air) were determined from XRD spectra (Bruker D8, Germany). XRD angular step is 0.01 degree 1 degree/min.

\section{Results and discussion}

\section{EBSPs}

Figure 3 shows SEM images of single phase STN94 and 8YSZ and example EBSPs collected at $10 \mathrm{kV}$ and $30 \mathrm{kV}$. Figure 4 (a) shows an SEM image of STN-YSZ 50\% (noxal9) and its sample EBSPs of STN phase and YSZ phase collected at $10 \mathrm{kV}$ and $30 \mathrm{kV}$ and (b) are SEM images of STNYSZ 10\% (air) and its sample EBSPs of the STN and YSZ phases collected at $10 \mathrm{kV}$ and $30 \mathrm{kV}$. Electron contamination marks in the SEM images reveal the collection positions of EBSPs on each sample. In the SEM images of Figure 4 the dark phase is YSZ and the bright phase is STN due to its electronic conductivity. For reference Figure 5 shows EBSPs of standard Si sample acquired at 10 $\mathrm{kV}$ and $30 \mathrm{kV}$. Figure 6 (a) is an example of an indexed EBSP (SEM $30 \mathrm{kV}$ ) of STN in STN-YSZ 10\% (air) with five selected bands and their Miller indices and (b) is the EBSP collected at the same location of (a) and collected at SEM $10 \mathrm{kV}$ which is used for measuring bandwidth. 


\section{Lattice constant measurement from XRD}

Figure 7(a) shows XRD peaks of the STN-YSZ 10\% (air) and (b) is that of STN-YSZ 50\% (noxal9). The peaks were compared to standard XRD peaks of STN and YSZ to identify their structure. XRD peaks in Figure 7(b) shows that both STN and YSZ phases in STN-YSZ 50\% (noxal9) have cubic lattices. In STN-YSZ 10\% (air) XRD peaks of STN indicated that this phase is cubic. However, XRD peaks of YSZ in STN-YSZ 10\% (air) shows that both cubic YSZ and tetragonal YSZ existed in the sample. In addition some unidentified peaks appeared labelled as red "u” in Figure 7(a). Measuring lattice constants from the XRD peaks was performed by using STOE WinXPow 2.20 software. The lattice constants of the cubic STN and YSZ are listed in Table 4 while the lattice constants of tetragonal YSZ are $a=3.6277 \AA \quad c=5.145 \AA$ and its space group is $\mathrm{P} 4_{2} / \mathrm{nmc}$. Although most refinements were performed to a precision of $10^{-5} \AA$, we conservatively list our experimental values to $10^{-4} \AA$ in Table 4. The collected EBSPs of YSZ in STN-YSZ 10\% (air) are all tetragonal. As this work is limited to cubic systems, the lattice constants of the tetragonal YSZ in this sample was not analysed.

\section{Calibration constant}

Five selected Kikuchi bands from the Si single crystal were used for computing the average calibration constant. Table 3 lists the Miller indices, the band width in pixels and the corresponding determined calibration constants. The calibration constants were computed using equation 6 resulting in a value of $C=164.0$ pixels. $\AA$

\section{Lattice constants Measured from EBSPs}

The lattice constants of cubic STN and cubic YSZ in the four samples were calculated using equation 6 and the results are listed in Table 4. Each calculation result in the table is an average lattice constant measured from 25 Kikuchi bands (5 bands x 5 EBSPs). The precision of the lattice constant measurement is determined by measuring standard deviation (SD) while accuracy is the measurement deviation between the EBSD determined lattice constants and those measured by XRD or from 
literature. Standard deviations of the measuring are in the range 0.03-0.04 $\AA$ and indicate the precision or consistency level of the calculation procedure. From XRD measurements, the lattice constant difference of STN in different samples is in the range 0.01-0.02 $\AA$ while lattice constant difference of YSZ is approximately $0.02 \AA$. With this precision, the lattice constant measured from EBSPs in this experiment cannot differentiate the differences in lattice constant of STN or YSZ between the four samples. The lattice constant accuracy, the last column of Table 4, of cubic YSZ is in the range 0.57-1.12 \% which is slightly larger the error observed for cubic STN, 0.19-0.35\%. Kikuchi band widths used in the calculation of STN lattice constant are in the range 185-207 pixels while those of YSZ are in the range 137-165 pixels. As such, this indicates that the lattice constant accuracy determined from EBSPs is dependent on the lattice constant itself. Thus to minimize the measurement error a set of widest detectable Kikuchi bands should be selected for the measurement.

Since recorded EBSPs are a discrete image of the real diffraction pattern, the precision of lattice constant measurement from EBSP is limited by the back projected image resolution which in turn is a function of the detector pixel size. Therefore, the precision of measuring lattice spacing is quantized in units of back projected pixels during band width measurements. As one lattice constant can be computed from various band widths wider bands can give higher lattice constant resolution than narrow bands. To study the effect of band size on the measurement precision, the lattice constant per band width $\left(a / A_{h k l}\right)$ of band series ((100), (200),..,(800)) of STN YSZ and Si were calculated by using equation 6 and the real lattice constants in Table 4 and the calibration $C=164.0$ pixels. Å. Figure 8 plots the lattice constant per pixel as a function of the band widths determined from the calibration constant and the lattice constants listed in Table 4. The values of lattice constant per pixel imply a lower theoretical limit of the measurement precision of the configuration of the system used in this work. The graph shows that wider bands give a higher theoretical measurement precision but the gain in precision is nonlinear and diminishes with increasing band width and higher order crystal planes. In practice wide bands tend to have low intensity due to the crystallographically corrected scattering factor associated with narrow spaced lattice planes. Low band intensity bands therefore 
have a low signal to noise ratio relative to the background noise in the EBSP and as such are subject to additional error in the measured bandwidth that is independent of the image resolution discussed above. This error is of a statistical nature and is most likely randomly distributed. The main user controlled parameters affecting the error is the combination of EBSP detector exposure time, detector gain and the number of detector image frames averaged during pattern acquisition.

In this experiment the largest band we can observe and identify is the (224) band of STN. The average bandwidth of (224) bands (over 37 bands) is 205.08 pixels with standard deviation $\left(\sigma_{1}\right) 1.71$ pixels. Multiplying $\sigma_{1}$ with $0.026 \AA$ /pixels (the lattice constant per pixel for a band size of 205 pixels), yields the precision $0.044 \AA$. While the narrowest bands in this experiment are the (133) family of YSZ with average bandwidth (over 15 bands) 138.07 pixels and standard deviation 0.88 pixels. The precision of the smallest band is therefore correspondingly $0.038 \AA$. This example shows that in practice the narrower band yields higher precision of the lattice constant measurement. Thus the optimum condition of each experiment must be determined to obtain the highest precision. The graphs in Figure 8 indicate the theoretical limit of our experiment due to the EBSP image resolution. The graphs also illustrate the precision dependency of the lattice constant itself. The smaller lattice of STN generally generates wider Kikuchi bands than YSZ and Si in the same acquisition configuration. Consequently theoretical precision limit of STN is slightly better than the limit of YSZ and Si. Due to the precision limit of measuring lattice constants from EBSPs, the original aim to determine lattice constant differences of STN in the three samples could not be resolved.

Ram et al. have demonstrated an accuracy of $<0.1^{\circ}$ in Kikuchi band edge location by removing the deleterious effects of band intersections (Ram et al., 2014). In comparison if one takes the pixel size and detector distance used for the measured STN and YSZ results above, a simple calculation reveals that the pixel size at the source point subtends $\sim 0.05^{\circ}$. Thus standard deviations of $1-2$ pixels in band widths yield a surprisingly good precision given that the current work relies on simple averaging to minimise the imprecision in Kikuchi band width induced by intersections. 
To obtain improved precision increased EBSP image resolution (reduced CCD sensor pixel size) is needed. Using a longer detector distance and a lower SEM accelerating voltage can magnify Kikuchi band width, however longer acquisition time is then needed due to lower backscattered signal intensity. A consequence of longer detector distance though is the loss of capture angle, thus fewer bands within a pattern can be analysed and the lengths over which Kikuchi bands can be averaged is reduced. Thus a trade-off between resolution and sampling statistics will occur. Detector distance is also ultimately limited by SEM chamber size. Better sensitivity CCD sensors can enhance signal-tonoise ratio and thus will increase the statistical precision of the measurement. However improving image quality cannot improve the image resolution limit. Another way to improve the band width measurement precision is to implement curve fitting of the slope of row averaging intensity (in Figure 2). This allows possibility to identify band edges with sub-pixel accuracy which may able to give an order of magnitude more back projected pixel size resolution. Given the work of (Ram et al., 2014) it is expected that combining measurements of many averaged deconvoluted band widths could yield additional increases in lattice constant precision. Further developments to increase precision and simplify data acquisition and analysis procedures accompanied with unit cell space group determination methods (Dingley and Wright, 2009; Li and Han, 2015) would lead to a useful EBSP based local crystallographic analysis tool .

\section{Conclusion}

Lattice constants of cubic STN and cubic YSZ in STN94, 8YSZ, STN-YSZ 50\% (noxal9) and STN-YSZ 10\% (air) were measured from SEM $10 \mathrm{kV}$ EBSP of size 1344x1024 pixels. The lattice constant of STN in STN94 STN-YSZ 50\% (noxal9) and STN-YSZ 10\% (air) are $3.92 \AA 3.94 \AA$ and $3.93 \AA$ respectively. While lattice constants of YSZ in 8YSZ and STN-YSZ 50\% (noxal9) are $5.17 \AA$ and $5.18 \AA$ respectively. Statistical precision of the measurement is quantified by the standard deviation of band width measurements and is in the range 0.03-0.04 $\AA$. With the determined experimental precision it was not possible distinguish difference in the lattice constants of STN or YSZ in the four different samples. The accuracy of the measurement compared to lattice constants 
measured from XRD or literature is in the range $0.09-1.12 \%$. The theoretical precision of lattice constant measurement from EBSP is limited by EBSP image resolution. Lattice constant resolution (lattice constant per band width) increases as band width increases. Lattice constant resolution also depends on the lattice constant itself. Although wider bands theoretically yield greater precision, in practice low intensity of the wide band increase band width measurement error thus reduces the measuring precision. Possible methods to improve the measuring precision are: higher resolution and sensitivity EBSD detectors, increasing detector distance, lowering SEM voltage, using curve fitting for sub-pixel band edge determination and averaging of deconvoluted Kikuchi bands.

\section{Acknowledgements}

NS would like to thank Dr. Harvinder Singh Ubhi, formerly of Oxford Instruments for his help in optimising EBSD pattern quality for lattice constant measurement. NS also wishes to acknowledge the Thai government for financial support. We are grateful to B. R. Sudireddy and E.Abdellahi for the provision of samples and for specimen preparation.

\section{Appendix 1: Data sampling of EBSP by radial back projection}

To perform the radial back projection, EBSP images of resolution 1344 x 1024 pixels need to mapped back into its captured area of size $38 \times 28 \mathrm{~mm}$, which is the size of CCD detector, on the phosphor screen by the factor 38/1344 $=0.02827 \mathrm{~mm} /$ pixel. Given the origin of the system and $\mathrm{x}, \mathrm{y}$

$\mathrm{z}$ directions are as shown in Figure 9. $p c=\left(x_{p c}, y_{p c}, 0\right)$ is position of the pattern centre on the phosphor screen. The radius of the projecting sphere is the detector distance $D$ and centre at the sample position $S=\left(x_{p c}, y_{p c}, D\right) . c_{1}^{\prime}=\left(x_{c 1^{\prime}}, y_{c 1^{\prime}}, 0\right)$ and $c_{2}^{\prime}=\left(x_{c 2^{\prime}}, y_{c 2^{\prime}}, D\right)$ are manually selected end points of central line of a selected band on the phosphor screen. $c_{1}=\left(x_{c 1}, y_{c 1}, z_{c 1}\right)$ and $c_{2}=$ $\left(x_{c 2}, y_{c 2}, z_{c 2}\right)$ are projection of $c_{1}^{\prime}$ and $c_{2}^{\prime}$ on the sphere surface. The band's capturing area is defined as a rectangle with length $L=D \theta$ (the arc length $c_{1}-c_{2}$ ) and width $W$, manually set to cover the width of the selected band, on the sphere surface (see Figure 9 and Figure 10). $\theta$ is the angle between $\vec{r}_{1}$ 
and $\vec{r}_{2}$ and can be determined from dot product of the two vectors. $\vec{r}_{1}$ and $\vec{r}_{2}$ are constant vectors of magnitude $D$ point from $S$ to $c_{1}$ and $c_{2}$. To determine $\vec{r}_{1}$ and $\vec{r}_{2}$, two auxiliary vectors $\vec{r}_{1}^{\prime}$ and $\vec{r}^{\prime}{ }_{2}$ point from $S$ to $c_{1}^{\prime}$ and $c_{2}^{\prime}$ respectively are introduced.

$$
\begin{aligned}
& \vec{r}_{1}^{\prime}=\left(x_{c 1^{\prime}}-x_{p c}\right) \hat{e}_{x}+\left(y_{c 1^{\prime}}-y_{p c}\right) \hat{e}_{y}-D \hat{e}_{z} \\
& \vec{r}_{2}^{\prime}=\left(x_{c 2^{\prime}}-x_{p c}\right) \hat{e}_{x}+\left(y_{c 2^{\prime}}-y_{p c}\right) \hat{e}_{y}-D \hat{e}_{z}
\end{aligned}
$$

$\hat{e}_{x} \hat{e}_{y}$ and $\hat{e}_{z}$ are unit vectors point to positive $\mathrm{x} y$ and $\mathrm{z}$ directions respectively.

$\vec{r}_{1}$ and $\vec{r}_{2}$ point to the same direction of $\vec{r}_{1}^{\prime}$ and $\vec{r}_{2}^{\prime}$ respectively and can be determined by resizing the vector $\vec{r}_{1}^{\prime}$ and $\vec{r}_{2}^{\prime}$.

$$
\begin{aligned}
& \vec{r}_{1}=\frac{D}{\left|\vec{r}_{1}^{\prime}\right|} \vec{r}_{1}^{\prime} \\
& \vec{r}_{2}=\frac{D}{\left|\vec{r}_{2}\right|} \vec{r}_{2}^{\prime}
\end{aligned}
$$

The points $c_{1}$ and $c_{2}$ can be determined by translating the sample position $S$ with the vectors $\vec{r}_{1}$ and $\vec{r}_{2}$ respectively. Figure 10 illustrates the data sampling grid of the capturing area. The square grid size $\delta$ is $0.02827 \mathrm{~mm}$, the pixel size of the CCD sensor. The number of grid points along the $W$ direction is $M \sim W / \delta$ and along the $L$ direction is $N \sim L / \delta$. The grid points are indexed by $i$ and $j$ ( $0 \leq$ $i \leq M$ and $-\frac{N}{2} \leq j \leq \frac{N}{2}$ ).The data sampling procedure consists of two steps: 1 ) determining position of grid point $p(i, j)$ on the sphere surface and 2) reading the pixel intensity for the grid point $p(i, j)$ from the corresponding point $p^{\prime}(i, j)$ on the phosphor screen.

Given $\vec{r}(i, j)$ is the position vector of grid point $p(i, j)$ with respect to the sample position, the position of the grid point $p(i, j)$ can be determined by translating the sample position with the vector $\vec{r}(i, j)$. The vector $\vec{r}$ is used to scan the grid points over the capturing area. $\hat{e}_{L}(i, j)$ and $\hat{e}_{W}(i, j)$ are unit tangential vectors at the point $p(i, j)$ which point along the $L$ and $W$ curves respectively. The scanning starting at the point $c_{1}\left(p(0,0)=c_{1}\right)$ and value of $\hat{e}_{L}(0,0)$ and $\hat{e}_{W}(0,0)$ at this point are:

$$
\hat{e}_{W}(0,0)=\frac{\vec{r}_{1} \times \vec{r}_{2}}{\left|\vec{r}_{1}\right|\left|\vec{r}_{2}\right|}
$$




$$
\hat{e}_{L}(0,0)=\frac{\hat{e}_{W}(0,0) \times \vec{r}_{1}}{\left|\vec{r}_{1}\right|}
$$

The principal scanning direction is along the arc $c_{1}-c_{2}$. Scanning in the $W$ direction is separated into two areas, positive $j$ and negative $j$. For positive $j$, moving from grid point $p(i, j)$ to $p(i, j+1)$ can be done by shifting the vector $\vec{r}(i, j)$ by $\delta$ to $\hat{e}_{W}(i, j)$ direction as in equation (13)

$$
\vec{r}(i, j+1)=\vec{r}(i, j)+\delta \hat{e}_{W}(i, j)
$$

For negative $j$, moving from grid point $p(i, j)$ to grid point $p(i, j-1)$ can be done in the same fashion.

$$
\vec{r}(i, j-1)=\vec{r}(i, j)-\delta \hat{e}_{W}(i, j)
$$

Assuming that $\hat{e}_{L}$ has not changed direction while moving between grid points along $\mathrm{W}$ e.g. $\hat{e}_{L}(i, j+1)=\hat{e}_{L}(i, j-1)=\hat{e}_{L}(i, 0)$ the new value of $\hat{e}_{W}$ at the points $p(i, j+1)$ and $p(i, j-1)$ can be calculated by using equations (9) and (10)

$$
\begin{aligned}
& \hat{e}_{W}(i, j+1)=\frac{\vec{r}(i, j+1) \times \hat{e}_{L}(i, 0)}{|\vec{r}(i, j+1)|} \\
& \hat{e}_{W}(i, j-1)=\frac{\vec{r}(i, j-1) \times \hat{e}_{L}(i, 0)}{|\vec{r}(i, j-1)|}
\end{aligned}
$$

After scanning the complete line $i$, the position vector $\vec{r}$ is moved back to $\vec{r}(i, 0)$ and the position is then shifted to the next line $(i+1)$ by $\delta$ in the $\hat{e}_{L}$ direction as in equation (11). The new vector $\hat{e}_{L}(i+1,0)$ also needs to be calculated using equation (12)

$$
\begin{aligned}
& \vec{r}(i+1,0)=\vec{r}(i, 0)+\delta \hat{e}_{L}(i, 0) \\
& \hat{e}_{L}(i+1,0)=\frac{\left[\hat{e}_{W}(i, 0) \times \vec{r}(i+1,0)\right]}{|\vec{r}(i+1, j)|}
\end{aligned}
$$

The scanning pattern is a fish bone structure as shown in Figure 10. Assuming vector components of $\vec{r}$ at the grid point $p(i, j)$ is $\vec{r}(i, j)=X_{i j} \hat{e}_{x}+Y_{i j} \hat{e}_{y}+Z_{i j} \hat{e}_{z}$ a vector pointing in the same direction but ending at the phosphor screen is $\vec{r}^{\prime}(i, j)=\frac{D}{Z_{i j}}\left(X_{i j} \hat{e}_{x}+Y_{i j} \hat{e}_{y}+Z_{i j} \hat{e}_{z}\right)$. The vector 
$\vec{r}^{\prime}(i, j)$ points to the projection point $p^{\prime}(i, j)=\left(\frac{D}{Z_{i j}} X_{i j}, \frac{D}{Z_{i j}} Y_{i j}\right)$ of the point $p(i, j)$ then pixel intensity of the point $p(i, j)$ is read from the point $p^{\prime}(i, j)$ 


\section{References}

Cullity, B.D., Stock, S.R., 2001. Elements of X-ray diffraction, 3. ed., internat. ed. ed. Pearson/Prentice Hall, Upper Saddle River, NJ.

Day, A.P., 2008. Spherical EBSD. J. Microsc. 230, 472-486. doi:10.1111/j.1365-2818.2008.02011.x

Dingley, D.J., Wright, S.I., 2009. Determination of crystal phase from an electron backscatter diffraction pattern. J. Appl. Crystallogr. 42, 234-241. doi:10.1107/S0021889809001654

Furrer, A., Strassle, T., Mesot, J., 2009. Neutron scattering in condensed matter physics, Series on neutron techniques and applications. World Scientific Pub, Singapore ; River Edge, N.J.

Karczewski, J., Riegel, B., Gazda, M., Jasinski, P., Kusz, B., 2010. Electrical and structural properties of Nb-doped SrTiO3 ceramics. J. Electroceramics 24, 326-330. doi:10.1007/s10832-0099578-7

Keller, R.., Roshko, A., Geiss, R.., Bertness, K.., Quinn, T.., 2004. EBSD measurement of strains in GaAs due to oxidation of buried AlGaAs layers. Microelectron. Eng. 75, 96-102. doi:10.1016/j.mee.2003.11.010

Kelly, A., Groves, G.W., Kidd, P., 2000. Crystallography and crystal defects /: A. Kelly, G.W. Groves, and P. Kidd, Rev. ed. ed. Wiley, Chichester; New York.

Koch, E., 2006. Application to the crystal systems, in: Prince, E. (Ed.), International Tables for Crystallography Volume C: Mathematical, Physical and Chemical Tables, International Tables for Crystallography. Springer Netherlands, pp. 6-9.

Li, L., Han, M., 2015. Determining the Bravais lattice using a single electron backscatter diffraction pattern. J. Appl. Crystallogr. 48, 107-115. doi:10.1107/S1600576714025989

Michael, J.., Eades, J.., 2000. Use of reciprocal lattice layer spacing in electron backscatter diffraction pattern analysis. Ultramicroscopy 81, 67-81. doi:10.1016/S0304-3991(99)00119-9

Page, K., Kolodiazhnyi, T., Proffen, T., Cheetham, A.K., Seshadri, R., 2008. Local Structural Origins of the Distinct Electronic Properties of Nb-Substituted SrTiO 3 and BaTiO 3. Phys. Rev. Lett. 101. doi:10.1103/PhysRevLett.101.205502

Ram, F., Zaefferer, S., Raabe, D., 2014. Kikuchi bandlet method for the accurate deconvolution and localization of Kikuchi bands in Kikuchi diffraction patterns. J. Appl. Crystallogr. 47, 264275. doi:10.1107/S1600576713030446

Saowadee, N., 2014. EBSP-Geo [WWW Document]. GitHub. URL http://github.com/jacobbowen/EBSP-Geo (accessed 9.5.16).

Saowadee, N., 2013. Two and three dimensional electron backscattered diffraction analysis of solid oxide cells materials (PhD Thesis). DTU Energy Conversion, Roskilde, Denmark.

Saowadee, N., Agersted, K., Ubhi, H.S., Bowen, J.R., 2013. Ion beam polishing for three-dimensional electron backscattered diffraction. J. Microsc. 249, 36-40. doi:10.1111/j.13652818.2012.03677.x

Schwartz, A.J. (Ed.), 2009. Electron backscatter diffraction in materials science, 2nd ed. ed. Springer, New York.

Sudireddy, B.R., Agersted, K., 2014. Sintering and Electrical Characterization of La and Nb Codoped SrTiO3 Electrode Materials for Solid Oxide Cell Applications. Fuel Cells 14, 961-965. doi:10.1002/fuce.201400017

Többens, D.M., Stüßer, N., Knorr, K., Mayer, H.M., Lampert, G., 2001. E9: The New HighResolution Neutron Powder Diffractometer at the Berlin Neutron Scattering Center. Mater. Sci. Forum 378-381, 288-293. doi:10.4028/www.scientific.net/MSF.378-381.288

Wilkinson, A.J., 1996. Measurement of elastic strains and small lattice rotations using electron back scatter diffraction. Ultramicroscopy 62, 237-247. doi:10.1016/0304-3991(95)00152-2

Wilkinson, A.J., Meaden, G., Dingley, D.J., 2006. High-resolution elastic strain measurement from electron backscatter diffraction patterns: New levels of sensitivity. Ultramicroscopy 106, 307313. doi:10.1016/j.ultramic.2005.10.001 
Winkelmann, A., Nolze, G., 2015. Chirality determination of quartz crystals using Electron Backscatter Diffraction. Ultramicroscopy 149, 58-63. doi:10.1016/j.ultramic.2014.11.013

Wright, S.I., Adams, B.L., 1991. Automated Lattice Orientation Determination From Electron Backscatter Kikuchi Diffraction Patterns. Textures Microstruct. 14, 273-278. doi:10.1155/TSM.14-18.273

Yashima, M., Sasaki, S., Kakihana, M., Yamaguchi, Y., Arashi, H., Yoshimura, M., 1994. Oxygeninduced structural change of the tetragonal phase around the tetragonal-cubic phase boundary in $\mathrm{ZrO} \quad 2 \quad-\mathrm{YO} \quad 1.5$ solid solutions. Acta Crystallogr. B 50, 663-672. doi:10.1107/S0108768194006257 


\section{Figures}

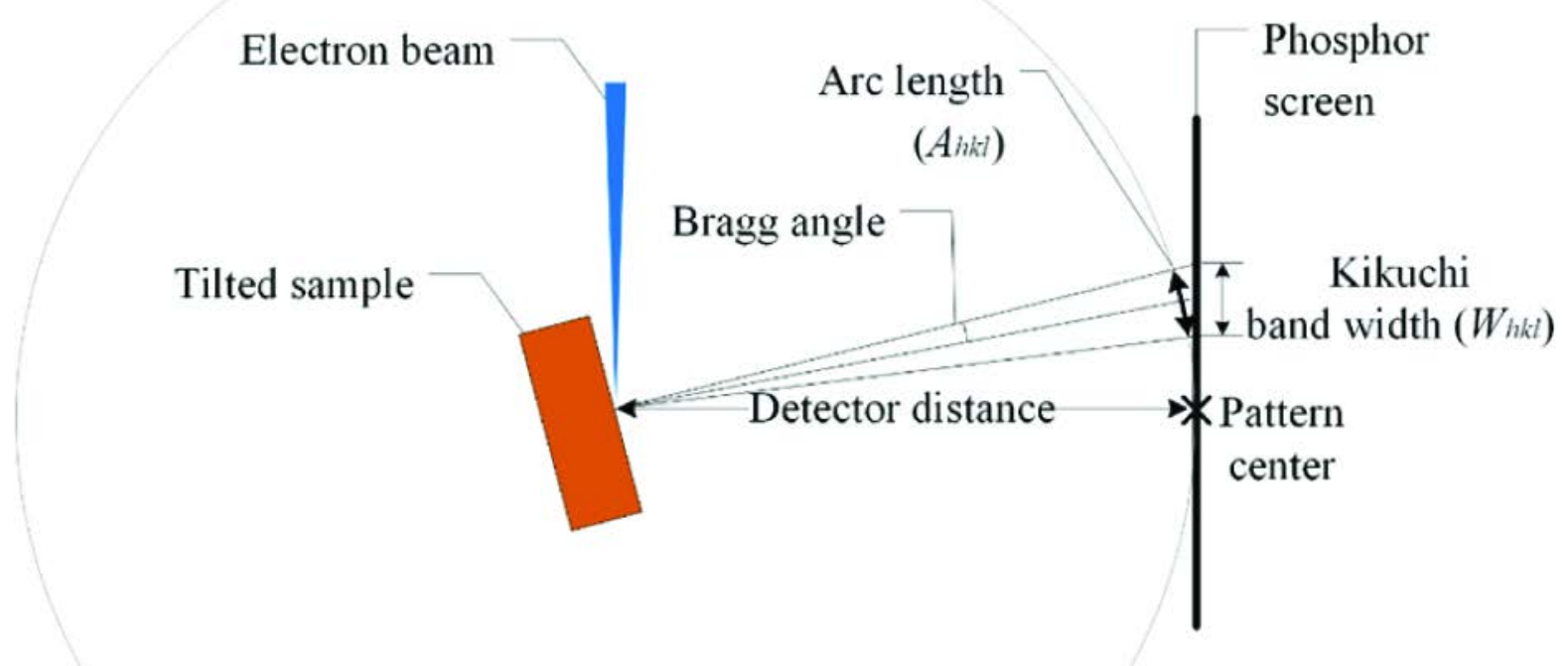

Figure 1: Schematic diagram of the relationship between Kikuchi bandwidth $\left(W_{h k l}\right)$ Bragg angle $(\theta)$ and the arc length $\left(\mathrm{A}_{\mathrm{hkl}}\right)$, real Kikuchi band width, which is a radial back projection of $W_{h k l}$ on the spherical surface of radius of detector distance.

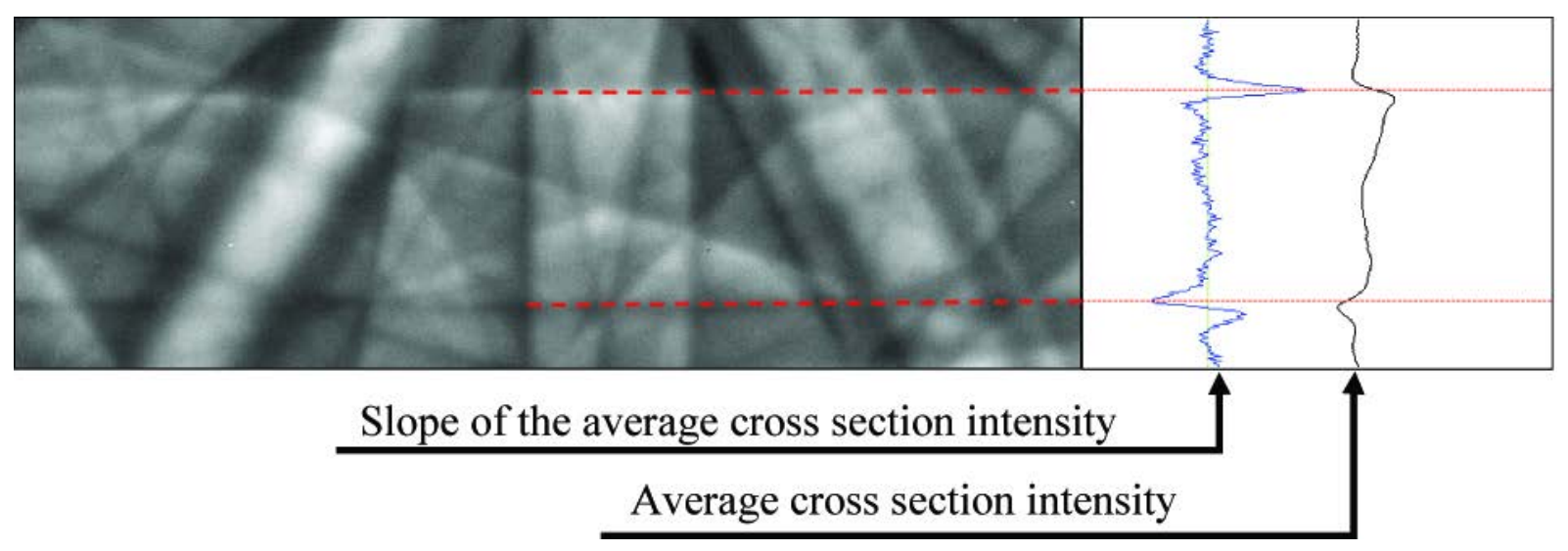

Figure 2: Row averaging of a captured band yields its average cross section intensity. The slope of the average band cross section intensity was used to determine the real band width $A_{h k l}$. 


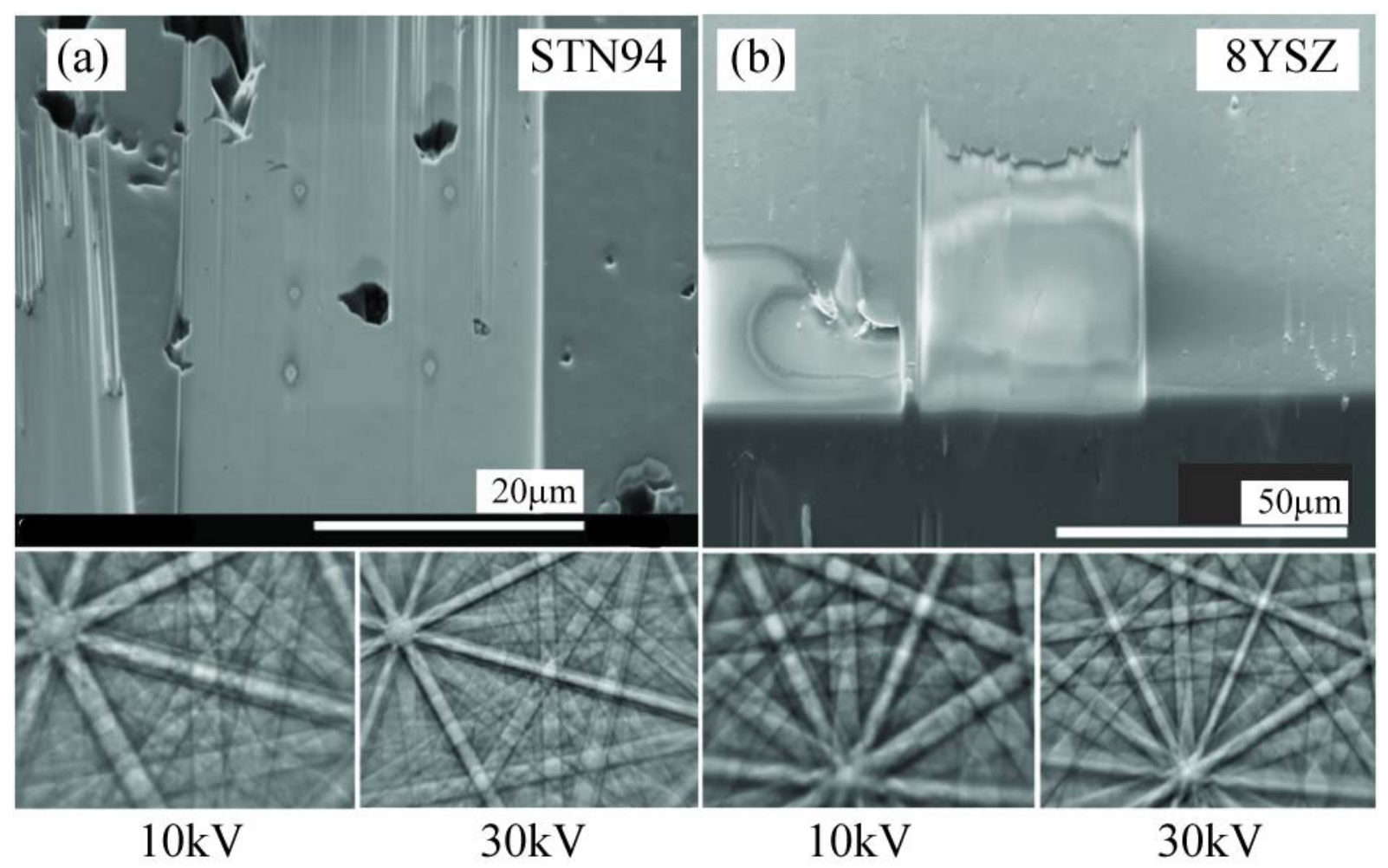

Figure 3: (a) SEM image of STN94 and its example EBSPs collected at $10 \mathrm{kV}$ and $30 \mathrm{kV}$ and (b) SEM image of YSZ and its example EBSPs collected at $10 \mathrm{kV}$ and $30 \mathrm{kV}$. Note: vertical features in SEM image indicate the surface area polished by FIB milling. 


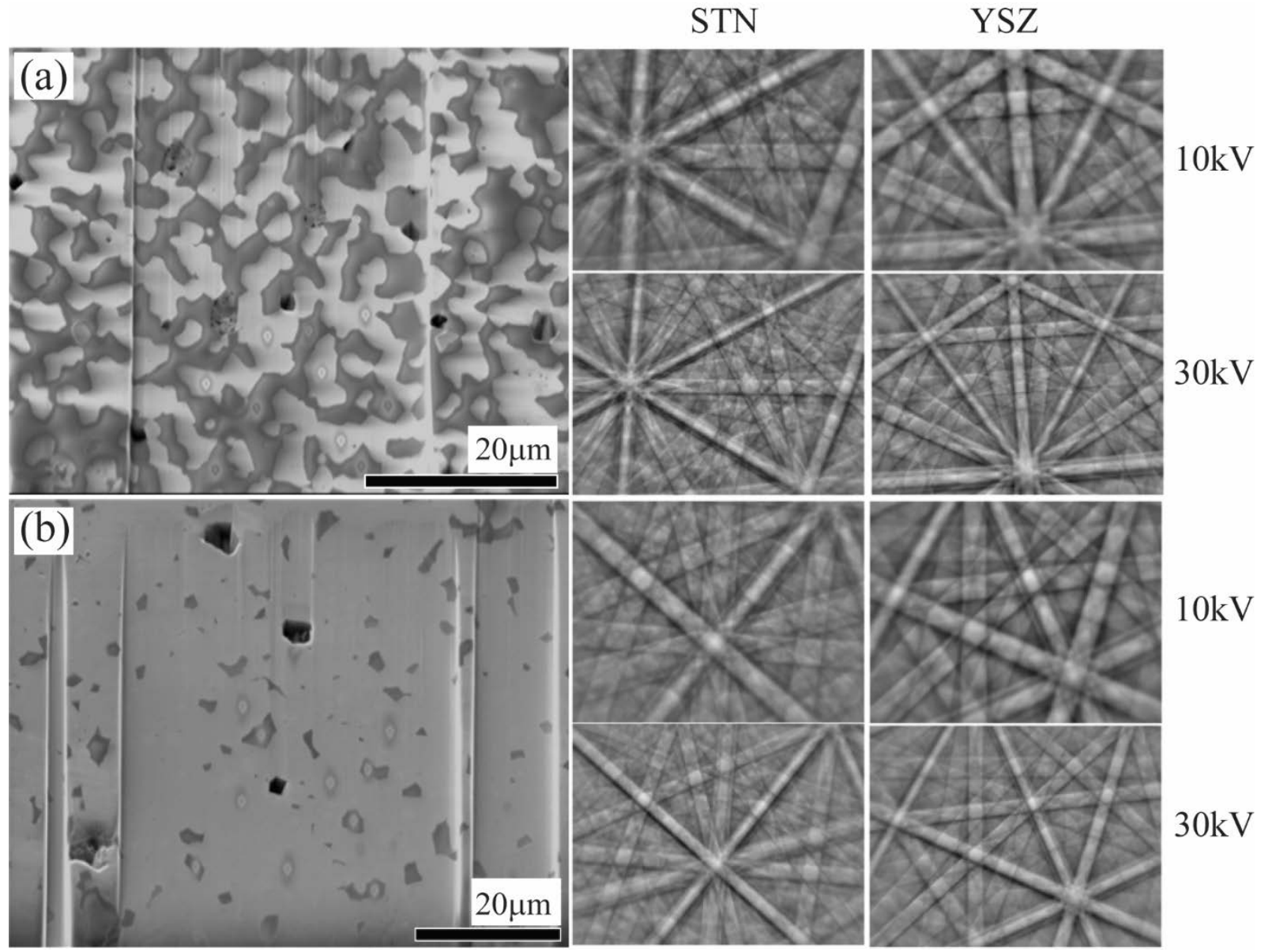

Figure 4: (a) SEM image of STN-YSZ 50\% (noxal9) and its example EBSPs of STN phase and YSZ phase collected at $10 \mathrm{kV}$ and $30 \mathrm{kV}$. (b) SEM image of STN-YSZ 10\% (air) and its example EBSPs of STN phase and YSZ phase collected at $10 \mathrm{kV}$ and $30 \mathrm{kV}$. 


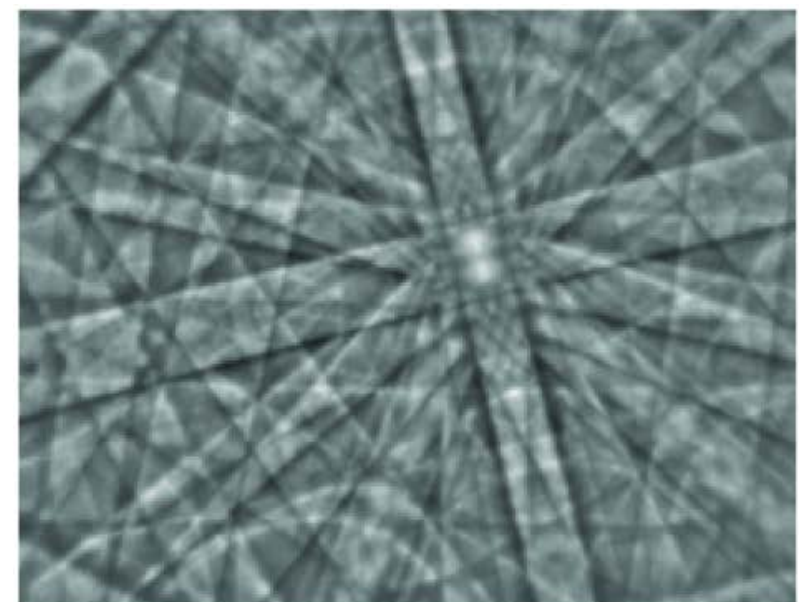

Si $10 \mathrm{kV}$

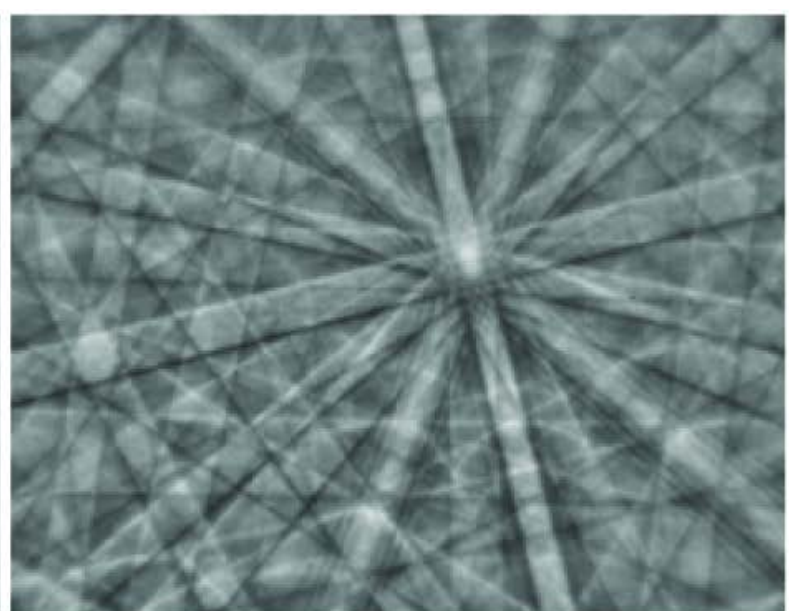

Si $30 \mathrm{kV}$

Figure 5:EBSPs of standard Si sample collected at SEM a) $10 \mathrm{kV}$ and b) $30 \mathrm{kV}$. 


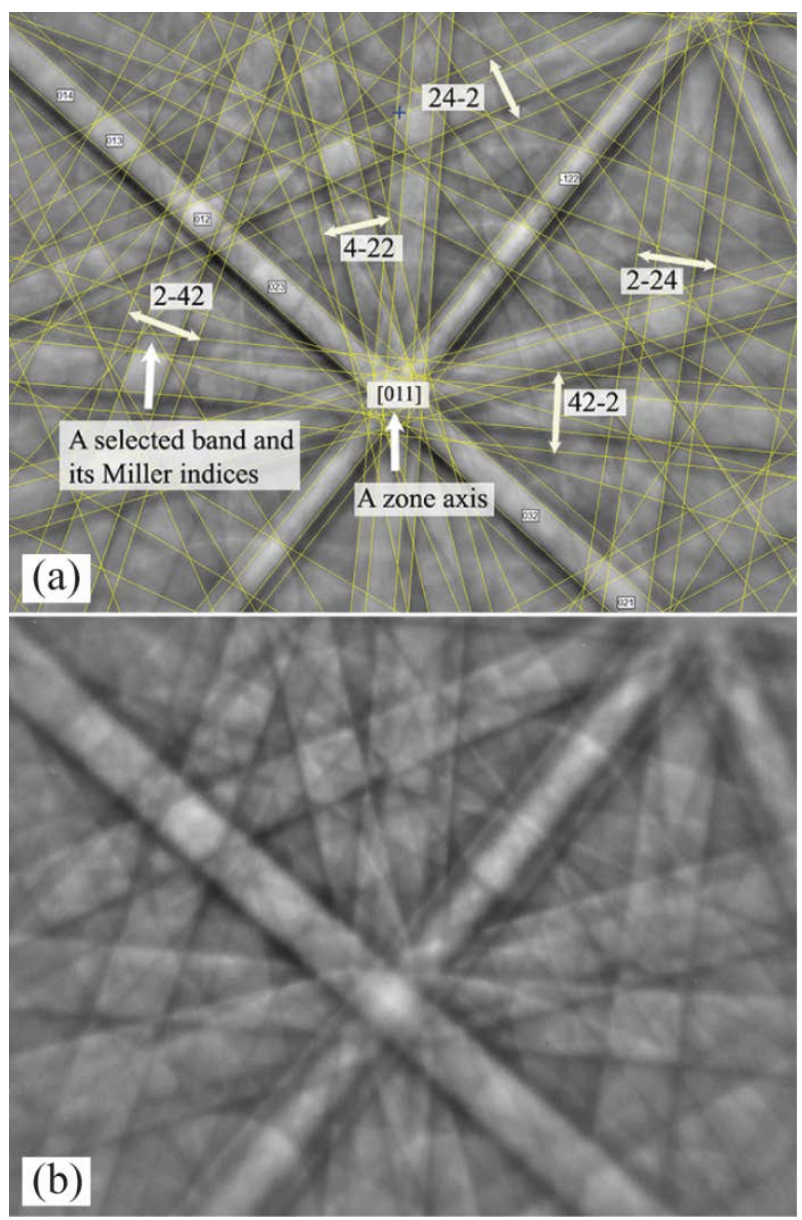

Figure 6: (a) An example of an indexed EBSP (30 kV) of STN in STN-YSZ 10\% (air) with five selected bands and their Miller indices. (b) The pattern collected at the same location of (a) but collected at $10 \mathrm{kV}$. 


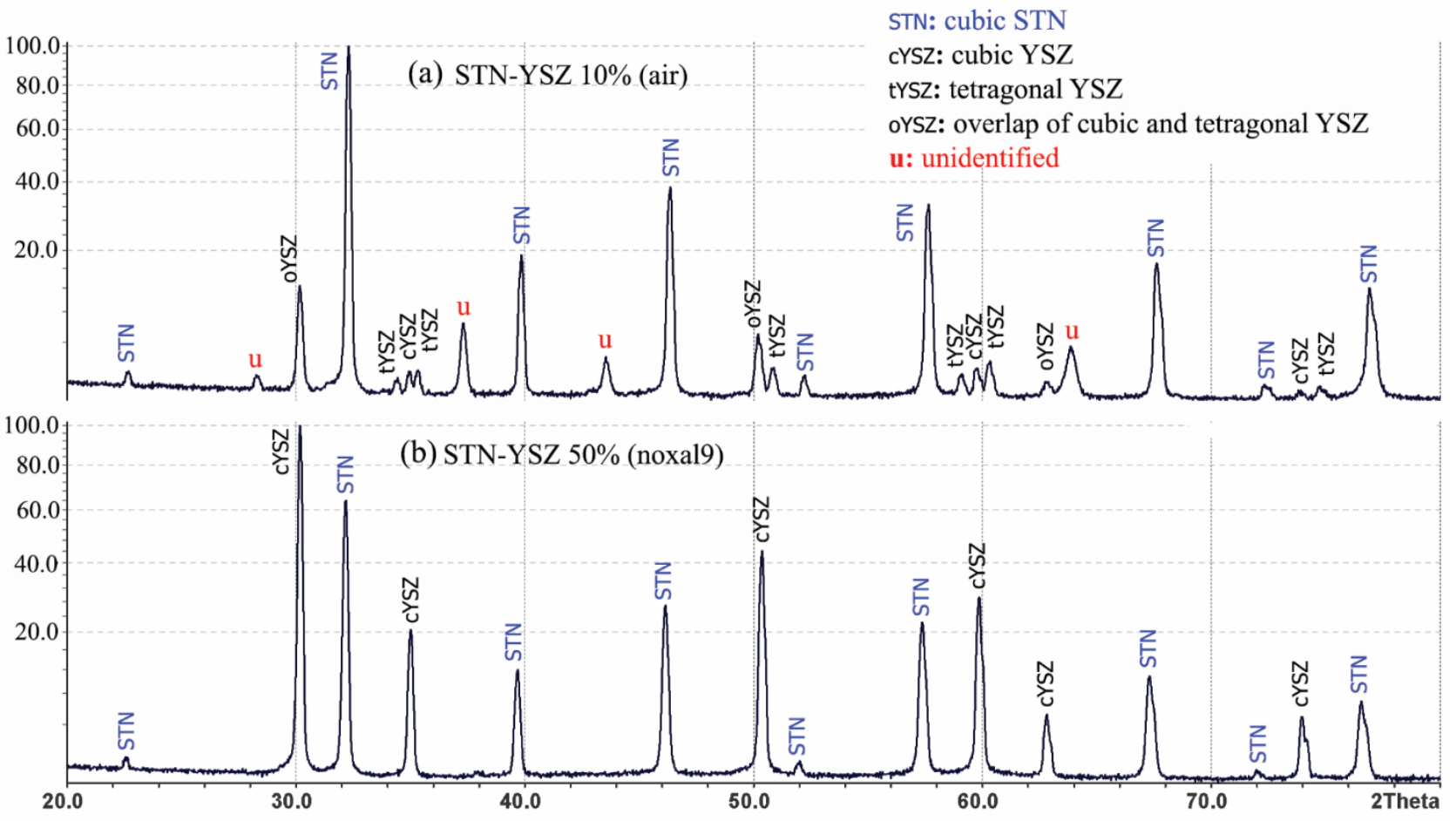

Figure 7: (a) is $2 \theta$ plot of STN-YSZ 10\% (air) XRD peaks and (b) is $2 \theta$ plot of STN-YSZ $50 \%$ (noxal9) XRD peaks. The STN phase in both samples is cubic. YSZ in STN-YSZ 50\% (noxal9) is cubic while both cubic and tetragonal YSZ existed in STN-YSZ 10\% (air). Unidentified STN-YSZ $10 \%$ (air) peaks are marked as “u” in red. 


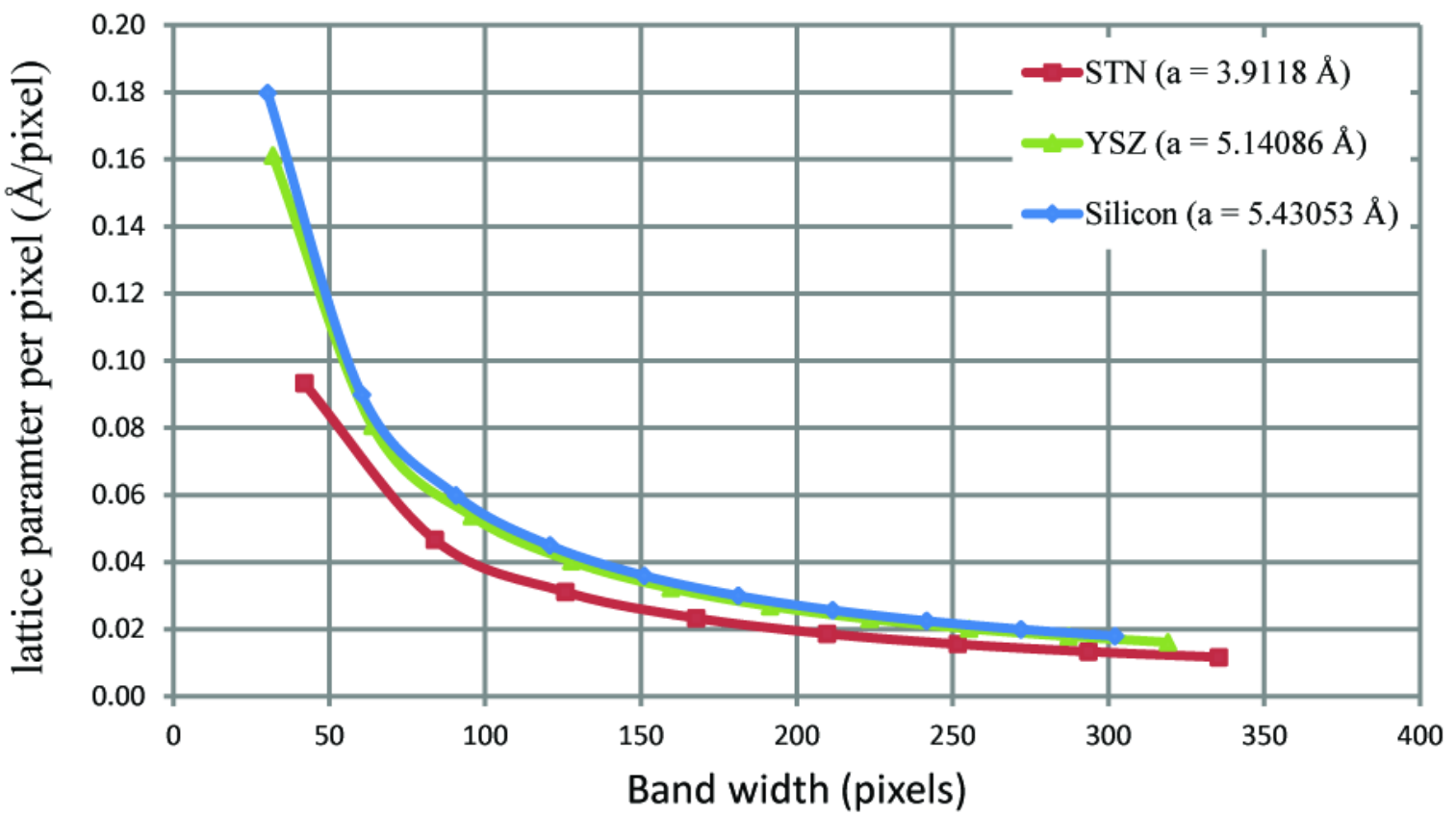

Figure 8: Plots of calculated lattice constant per pixel $a / A_{h k l}$ of various band widths of Si, STN and YSZ. 


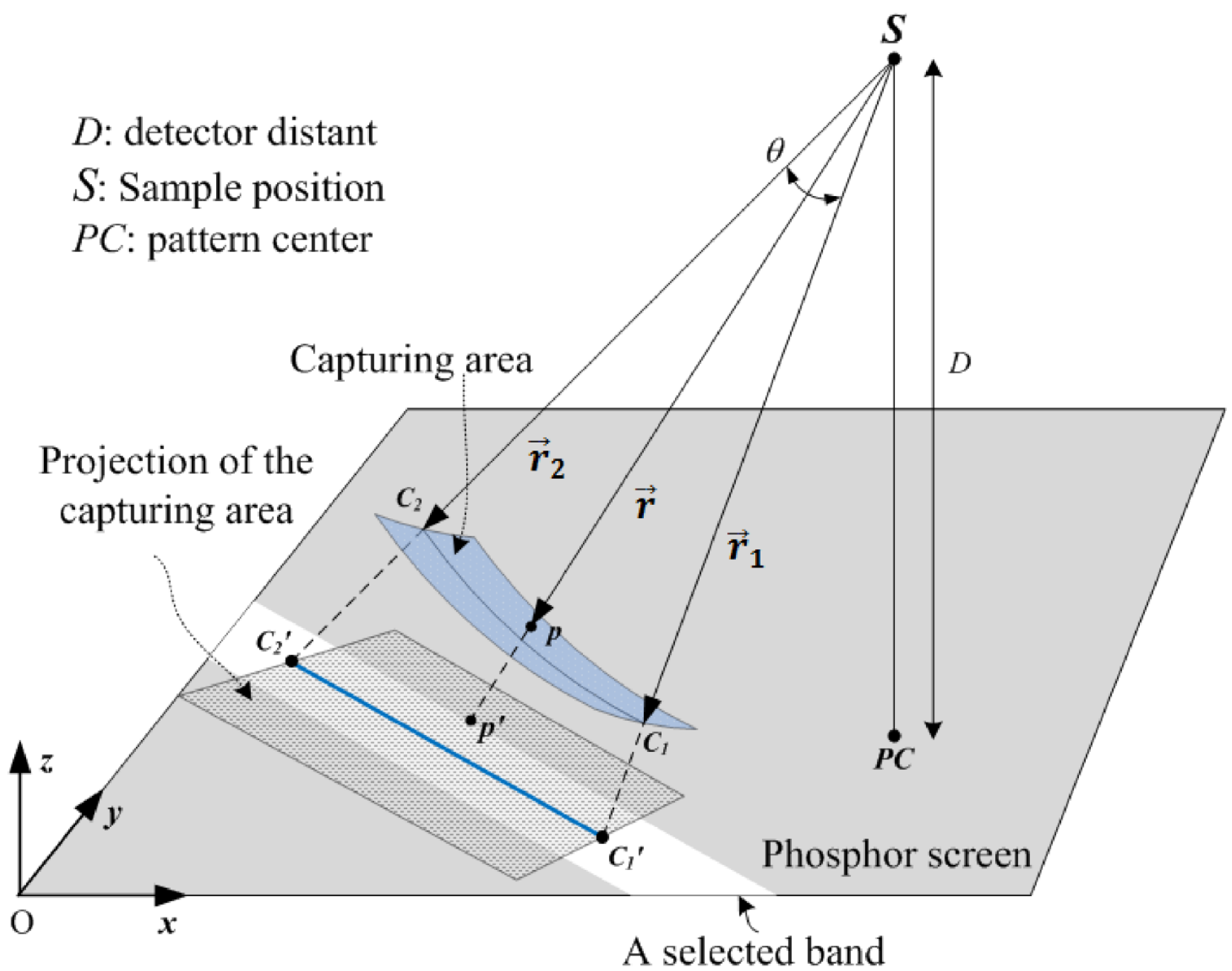

Figure 9: Schematic shows the radial back projection system. The centre of the projecting sphere of radius $D$ is at the sample position $S . c^{\prime}{ }_{1}$ and $c_{2}^{\prime}$ are selected end points of the central line of a selected band and $c_{1}$ and $c_{2}$ are their projection on the sphere surface. 


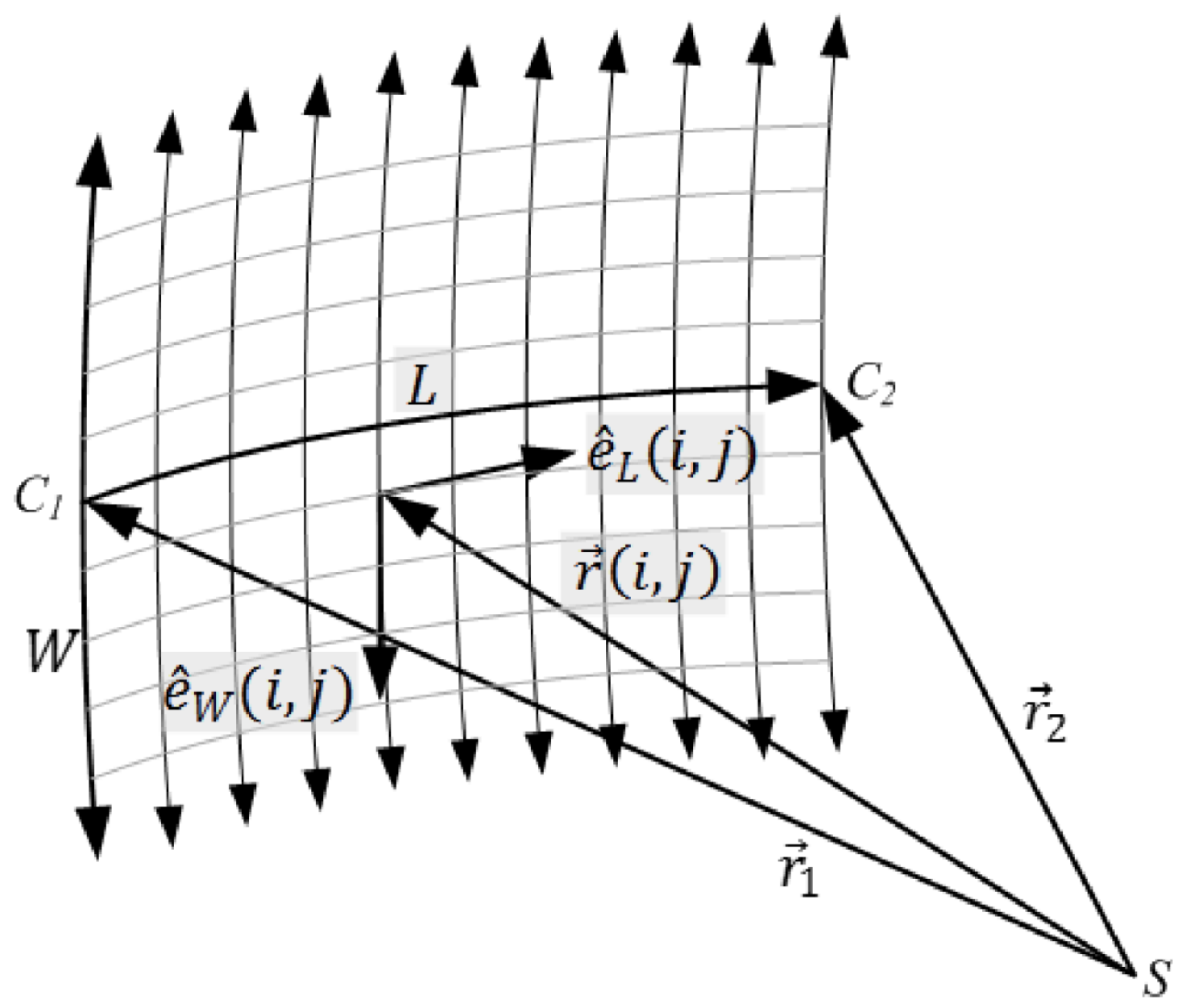

Figure 10: Data sampling grid of the capturing area on the sphere surface. 
Table Captions

Table 1: Summary of Samples.

\begin{tabular}{|l|l|l|}
\hline Samples & Phase ratio (STN:YSZ) & Sintering atmosphere \\
\hline STN94 & $1: 0$ & air \\
\hline 8YSZ & $0: 1$ & air \\
\hline STN-YSZ 50\% noxal9 & $0.5: 0.5$ & 9 vol\% $\mathrm{H}_{2}$ and $91 \mathrm{vol} \% \mathrm{Ar}$ \\
\hline STN-YSZ 10\% air & $0.9: 0.1$ & air \\
\hline
\end{tabular}

Table 2: Summary of EBSP collection.

\begin{tabular}{|c|c|c|c|}
\hline \multirow{2}{*}{ Sample } & \multirow{2}{*}{ Phases } & \multicolumn{2}{|c|}{ Number of patterns } \\
\hline & & SEM $30 \mathrm{kV}$ & SEM $10 \mathrm{kV}$ \\
\hline \multirow[t]{2}{*}{ STN-YSZ 50\% (noxal9) } & STN & 5 & 5 \\
\hline & YSZ & 5 & 5 \\
\hline \multirow[t]{2}{*}{ STN-YSZ 10\% (air) } & STN & 5 & 5 \\
\hline & YSZ & 5 & 5 \\
\hline STN94 & STN & 5 & 5 \\
\hline 8YSZ & YSZ & 5 & 5 \\
\hline Silicon & $\mathrm{Si}$ & 1 & 1 \\
\hline
\end{tabular}


Table 3: Calibration constant computed from five Kikuchi bands in single crystal Si.

\begin{tabular}{|l|l|l|}
\hline $\begin{array}{l}\text { Bands } \\
(h k l)\end{array}$ & $\begin{array}{l}\text { Band width } \\
\text { (pixels) }\end{array}$ & $\begin{array}{l}\text { Calibration constant } \\
\text { (pixels. A })\end{array}$ \\
\hline$(224)$ & 150 & 166.2 \\
\hline$(2-42)$ & 149 & 165.1 \\
\hline$(2-4-2)$ & 148 & 164.1 \\
\hline$(026)$ & 190 & 163.2 \\
\hline$(026)$ & 188 & 161.4 \\
\hline & & $164.0 \pm 1.8$ \\
\hline
\end{tabular}

Table 4: Average lattice constants of YSZ and STN phases in STN-YSZ 10\% (air) STN-YSZ 50\% (noxal9), STN94 and 8YSZ computed from EBSPs compared to XRD results.

\begin{tabular}{|c|c|c|c|c|}
\hline \multirow[b]{2}{*}{ Samples } & \multirow[b]{2}{*}{ Phases } & \multicolumn{2}{|c|}{ Lattice constant measured from } & \multirow[b]{2}{*}{ Accuracy } \\
\hline & & $\begin{array}{c}\text { EBSPs } \\
\text { Average } \pm \text { SD }\end{array}$ & XRD & \\
\hline $\begin{array}{l}\text { STN-YSZ } 10 \% \\
\end{array}$ & STN & $\mathrm{a}=3.93 \pm 0.04 \AA$ & $\mathrm{a}=3.9163 \AA$ & $0.35 \%$ \\
\hline (Air) & YSZ & No calculation & Undetermined & \\
\hline STN-YSZ $50 \%$ & STN & $\mathrm{a}=3.94 \pm 0.04 \AA$ & $\mathrm{a}=3.9324 \AA$ & $0.19 \%$ \\
\hline (noxal9) & YSZ & $a=5.18 \pm 0.03 \AA$ & $\mathrm{a}=5.1224 \AA$ & $1.12 \%$ \\
\hline STN94 & STN & $\mathrm{a}=3.92 \pm 0.03 \AA$ & $\mathrm{a}=3.9118 \AA$ & $0.21 \%$ \\
\hline 8YSZ & YSZ & $\mathrm{a}=5.17 \pm 0.03 \AA$ & $\begin{array}{l}\mathrm{a}=5.14086 \AA \\
\text { (YASHIMA et al., 1994) }\end{array}$ & $0.57 \%$ \\
\hline
\end{tabular}

